\title{
Post-stroke visual rehabilitation - the impact on life quality of the stroke survival patients - a brief review
}

\author{
Adriana Bulboaca ${ }^{1,2}$, Angelo Bulboaca ${ }^{2}$, Ioana Stanescu ${ }^{1,2}$, Mihai Blidaru ${ }^{1}$, Alexandra Ina Bulboaca ${ }^{1}$, \\ Cristina Nicula $^{1,3}$, Gabriela Dogaru ${ }^{1,2}$
}

Corresponding author: Ioana Stanescu, E-mail: ioanastane@yahoo.com,

\author{
1 "Iuliu Hatieganu" University of Medicine and Pharmacy, Cluj-Napoca, Romania \\ ${ }^{2}$ Clinical Rehabilitation Hospital, Cluj-Napoca, Romania \\ ${ }^{3}$ Ophthalmology Clinic, Cluj-Napoca, Romania
}

\begin{abstract}
Visual rehabilitation therapy (VRT) is one of the most important and difficult post stroke rehabilitation component that can considerably improve the quality of patient's life. There are also evidences that VRT contribute to a better motor recuperation, by offering a support for spatial and temporal orientation due to process of motor activity recuperation. Visual rehabilitation methods can be classified into the following groups: Visual Substitution Therapy, Visual Scanning Training (VST), Audio-Visual Scanning Training (AViST) and Vision Restoration Training (VRT). Even the evaluation of the results of training methods have an important subjective component, being assessed by various questionnaires, there are continues improving of these methods designed to achieve better results in post stroke rehabilitation therapy. Understanding the anatomical support and psysiological background of the visual process in brain networks, and also, the pathophysiology of visual function impairment after stroke, could be a good support for an personalized rehabilitation therapy adapted to each patient. This paper is aimed to emphasise the main methods of vision rehabilitation therapy in order to offer to the post stroke rehabilitation team an instrument for their day by day activities designed to improve the quality of life for stroke surviving patients.
\end{abstract}

Key words: stroke, visual impairment, visual rehabilitation, neuroplasticity, hemianopsia, diplopia,

\section{Introduction}

The World Health Organization (WHO) provides a specific definition for low vision as follows: impairment of visual function even after standard correction of refractive errors or specific treatments (medical or surgical) with visual acuity less than $6 / 18$ (3/10 on Snellen chart assessment) or a visual field with concentric reduction at less than 10 degree from the fixation point, or the inability to use the vision for daily tasking execution or planning activities $(1,2)$. According with WHO, visual disability classified by visual acuity grade can be represented by three categories: 1: moderate visual impairment (VA between 3/10 and 1/10), 2: severe visual impairment (VA between 1/10 and 1/20), 3: blindness (VA < 1/20) (1). According with visual field (VF) defect there are: moderate visual impairment (VA field restricted between 10 and 20 degrees), severe visual impairment (VF restricted between 10 and 5 degrees), and blindness (VF restricted to less than 5 degrees (1). According with orientation and mobility ability that is subjective, but influencing daily activities, there are also moderate impairment of vision when the patient is able to localize and avoid obstacles, severe visual impairment when the activity is restricted with aids, and blindness when the visual orientation is unreliable (patient is orientating by hearing) (1). One of the most important visual impairment is consequently to stroke, both ischemic or hemorrhagic stroke can lead to visual disabilities. Visual impairment after stroke is a frequent consequence in surviving patients and can affect approximately $60 \%$ of the patients (3). Where visual problems existed in patient's history, there is possible not to be immediately recognized as part of the stroke syndrome and attributed to other causes such are migraine with aura, where the visual disturbances can precede the migraine pain, due to their common pathophysiological pathways, or hypertensive crisis $(4,5)$. Visual impairment can consists in visual field loss, double vision, nystagmus, blurred vision (usually on the same side with motor deficit) perceptual and psychological problems (6). The visual system disorders are close related to stroke topography (7). The stroke survival patients are also complaining of dry eyes, symptom related to their age or to the lack of attention to a proper treatment of eye dryness (8). The consequences of impairing of visual function can be very various, from loosing of confidence due to increased risk for falls and accidents with trauma, to increased rehabilitation 
difficulties. Impact of visual problems on the quality of life after stroke it is also considerable (8). There are also serious difficulties to adapt to this new situation and despite of the patients efforts to reduce the visual impairment (by using a magnification glass, changing their posture, increasing light intensity or changing the distance to the visual target) they are often unable to increase the visual comfort by themselves. This article is designed to analyze the importance of identify the visual impairments consequently to a stroke, in surviving patients, and to systematize the patients clinical approaching, in order to improve their neurological rehabilitation. The aim of this paper is to enhance the main neurophysiologic processes that are the basis of visual perception and the most important rehabilitation therapies that can contribute to visual rehabilitation in stroke patients.

\section{Neurophysiologic basis of vision and associated deficits}

The human visual pathways that are implicated in perception, transmission and processing of the visual information are still under the study and the organizational principles within this region are not yet well understood. The integrity of visual pathways contribute to a correct decisional activity and constitute an important guidance for motor activity, properly integrated in time and space. Despite of the classical pattern of visual information transmission from retina to the occipital cortex, through the visual pathways via reticulo-geniculo route, there are alternative pathways for visual information transmissions that play an important role for vision rehabilitation due to neuroplasticity phenomenon (9). Modern neuroimaging techniques including functional magnetic resonance imaging (fMRI) and diffusion tensor MRI and fibre tractography (DTI) support the existence of an important circuitry for visual perception of words, colours, forms and faces in occipito-temporal cortex (10). There are other visual association cortex that contains a large number of specialized visual areas as are V4 specialized for colour perception or V5 area specialized for motion perception (11). Mainly, the information that are transmitted through extrastriatal cortex are directed along two pathways: ventral pathways (for forms recognition and colours), involving inferior temporal cortex and dorsal pathways (for spatial aspects), involving parietal cortex (12). This is highly relevant in understanding brain capacity to recover visual function in patients with brain lesions. These two pathways are complementary in environmental perception and essential for a spatial and temporal integration of the motor function $(11,13)$. According with visual field impairment there are several lesions that can result in specific changes of visual field assessed by perimetry: unilateral defects as are centro-cecal scotoma that can result from retinal ganglion cells lesions (papillo-macular bundle area), or amblyopia that can result from interruption of the neural connections between the eye and the visual pathways from various causes including intraorbitar and prechiasmatic segment of the optic nerve $(14,15)$. Hemianoptic, quadrianoptic and altitudinal defects that result from chiasmatic and post chismatic lesions of the visual pathways including occipital cortex (16). Visual integration disorders, associated with stroke, consist in failure of the visual cortical areas to process the visual information and can be classified as follow: alexia without agraphia associated with lesions of the corpus callosum (splenium) that connects the right occipital lobe and angular gyrus (patients in this situation are not able to read and failure to resolve their deficit by spectacle prescription) (17) ; visual agnosia that can be perceptive visual agnosia (the objects are not recognized and are associated with bilateral parieto-occipital lesions) and associative visual agnosia (where the visual perception of the objects can't be associated with any visual experience or object memory) (18) ; Gertsmann syndrome represented by finger agnosia associated with rightleft disorientation, agraphia and acalculia that occur dominance hemisphere lesions (inferior parietal lobe - angular gyrus) (19) ; colour agnosia associated with temporal associative cortex lesions (20).

Visual rehabilitation therapy can be supported by some residual structures that are typically spared by the damage: island of better vision in the blind field, visual field borders (at the level of visual field impairment), extrastriatal pathways spared by the damage, perilesional spared tissue and some high level neuronal networks preservation (15).

\section{Visual rehabilitation methods}

Patient's ability to perform visual tasks and potential benefits of rehabilitation are needed to be estimated before the decision to apply the visual rehabilitation therapy. Influencing factors as hearing loss, cognitive troubles, tremor, sensory or motor deficit and depressive syndrome are also need to be identified $(21,22)$. 
Visual rehabilitation methods can be adressed to the central vision restoration, peripheral field restoration or the restoration of the vision due to hemianopia (23). According with the topography of the lesions, focal lesions are associated mainly with hemianoptic or sectorial defect and the lesions are represented mainly by stroke, since peripheral/central field defects are mainly associated with multifocal lesions due to diseases as is multiple sclerosis (24). Since multiple sclerosis has a pattern of demyelisation or remyelisation, that are cyclic processes, it is difficult to assess vision restoration therapies because the disease itself is naturally associated in evolution with spontaneous visual rehabilitation due to remyelisation process. Therefore, permanent lesions produced in ischemic conditions, are the situation where the visual restoration techniques can contribute to the improvements in visual function. Visual rehabilitation methods follow 2 concepts represented by restoration of visual function and compensation of the classical pathways of visual system with alternative pathways and cortical areas (25). Compensatory and restorative approaches have become intensive studied in recent researches because of the continued expansion of studies that reported plastic reorganization in the human visual system (26).Visual rehabilitation methods can be classified into the following groups: Visual Substitution Therapy, Visual Scanning Training (VST), Audio-Visual Scanning Training (AViST) and Vision Restoration Training (VRT) $(25,27)$. The last method of rehabilitation, even if has the largest potential, is the most controversial. However, there are several factors that can impair visual rehabilitation therapies: fewer functional neurons in residual structures spared by the damage, less physiological functionality of these structures compared with main visual pathways, and failure to correct visual acuity by spectacles due to other concomitant eye conditions associated with patient age, as are optic nerve ischemia or age related macular degeneration (28).

Visual Substitution Therapy include refraction errors correction, prisms prescription, orthoptic exercises, low vision aids, and advice (29). Refraction error correction it is an important step towards the better visual acuity that is an essential contributor for the other rehabilitation methods applied to the patients. If the visual acuity is not the best possible after spectacle prescription, other training methods are not resulting in maximum rehabilitation. Prism prescription and orthoptic exercises designed to diplopia are only for visual comfort improvement but are also important for patient rehabilitation.

Visual Scanning Training is mostly addressed to compensatory mechanism of visual rehabilitation and consists in three types of exercise designed for compensatory pathways training : the first type of exercise based on visual search in which patients have to find one or more targets among distracters, second type based on finding a specific target that is not surrounded by distracters, and the third type when the targets are presented on the horizontal axes and the participants are making large and fast movements towards the target (30). Recent studies showed that VST applied to the patients with homonymous hemianopsia, due to postchiasmatic lesions, can specifically improves detection of peripheral visual stimuli and avoiding obstacles when moving around during mobility-related activities (31). Orientation difficulties after hemianoptic lesions have two different approach: rehabilitation of the deficitary visual field by visual stimulations and explorative eye movements in the direction of the hemianoptic field (32). Cerebral perception of visual information in cortical areas can be also affected, therefore, visual rehabilitation in hemianopsia can require and multidisciplinary team approaching (neurology and ophthalmology specialists) (32). Visual rehabilitation in visual field defect depends on surviving neurons and their topography on visual pathways and cortical area. Perceptual learning and receptive field plasticity also play an important role in vision restoration (33). Efficiency of VST is also in accordance with reaction time improvements, that indicate that the plasticity of temporal processing information can also contribute to a better rehabilitation of the vision (34). Functional imaging studies of the brain activity previous and after VST demonstrate the potential role of plasticity in vision rehabilitation therapy. The exact mechanism of this effect is still unknown, and evidence of visual improvement are only based on visual field assessment and subjective questionnaires. Despite that, a significant percentage of the patients reported a subjective improvement in daily activities, that is an important factor for life quality improvement (35). Mueller et al reported a notable subjective improvement in $70.9 \%$ of the patients, improvement that were independent of the lesion etiology and age, with most benefits for the patients older than 65 years 
and for the patients who had a preservation of larger areas of residual function at baseline (35). Their results were validated by visual field assessment at baseline and at the end of therapy and by questionnaires (36). More complex training by this technique consist in daily training in a driving simulator where the patients are able to develop saccadic eye movements as a compensatory mechanisms, for a better localisation of the target in space and time, because the stimuli succession are more rapid and the patient have to focus on their presentation (37). Therefore, saccadic behaviour can constitute and important phenomenon that can be trained in order to rehabilitate the visual field in patients with hemianopia. Combined with immediate visualization of gaze behaviour the method provide a feedback mechanism for a better treatment strategy. Clinical research in this area can improve the understanding of the pathophysiological mechanisms that are on the basis of visual improvement by VST, and eventually can contribute to scientific improvements of the method.

Audio-Visual Scanning Training (multisensory stimulation training) consists in audio-visual stimulation toward the visual field defect and is considered to be based on stimulation of superior colliculus that is an important central nervous system structure involved in initiation and execution of saccades. The mechanism underlying on this process is not entirely known but is leading to improvement of the accuracy and searching time during the visual exploration (38). Furthermore, gradually decreasing of the temporal interval between acoustic stimulation and visual stimulation by a visual target, was reported to improve the visual restoration after 1 month of therapy, $4 \mathrm{~h}$ a day, with a long lasting results, for those who had audio-visual stimulation, compared with the patients with only visual or audio stimulation, in hemianoptic patients. This showed that multimodal sensory stimulation can improve visual restoration therapy in patients with brain lesions (39). Besides of the visual field improvements in patients with hemianopsia due to brain lesions, there are a studies that demonstrated that audio-visual stimulation therapy can be also useful in patients with visuospatial attention deficit as are patients with neglect, improving multisensory integration. Audiovisual stimulation was failed to show an improvement in patients who presented both deficits (hemianopsia and neglect) $(40,41)$. The results were better if the audio and visual stimuli were aliniated in the same direction and were spatially closed (41). The visual function restoration is possible due to remapping processes of the cortex operating in higher-level visual perception areas of the parietal cortex offering a visual integration in time and space of the rapid successive of foveal retinal images and ensuring the environmental orientation by the spatial perception of the target (42). If the visual stimulus is associated with acoustic stimulus, the remapping process is enhanced due to cross-modal spatial integration between auditory and visual inputs that is an important phenomenon in space perception $(41,42)$. The explanation of the improvements due to AudioVisual Scanning Training technique is not yet completely understood some studies suggesting that multisensory integration and cross modal attention take place in different cortical areas that deserve visual function, while others show the involvement of early sensory specific areas (25). There are also studies that reported that multisensory cortical integration and attention are interacting, and are related to the lesion topography (43).

Vision Restoration Training. One of the most important component for visual function restoration is the improvement of visual stimuli discrimination together with the improvement of the ability to perceive various light intensity attributed to visual stimuli. Long term training (6 month of daily training) can improve the luminance sensitivity improvements in trained patients and perception area (44). Compensation and restoration are different forms of visual rehabilitation, and the difference between them consists in their different pathophysiological mechanisms: compensation is designed to recruit the alternative visual pathways and brain visual perception areas and restoration consist in stimulation of partially injured visual areas. Both of the process involve neuronal plasticity even there are serious controversies about the real possibility of restoration mechanism (45).

\section{Consequences of vision rehabilitation therapy}

In general terms, Visual Restoration Training aim at improving the magnitude of visual function, while Visual Scanning Training and Audio-Visual Scanning Training compensate for the visual field loss (25). While substantial spontaneous recovery can occur in the first few weeks to months brain lesions, cortical blind defects tend to become stable and permanent in a high percent of the patients (44). Most 
of the therapies targeting visual rehabilitation are focused on developing of compensatory mechanisms as are eyes movement strategies, or prism lens indication for diplopia (46). Patients also develop head adapted position in order to improve visual function position and to compensate visual field defect (47). The methods for visual function rehabilitation are still under research and subjective control of results (based mostly on visual acuity assessment, visual field assessment and questionnaires about visual improvement in daily activities) rise difficulties in clinical implementation of these therapies. Regardless of these concerns and of the lack of evidences about the mechanisms of visual rehabilitation therapies, the results of vision rehabilitation therapy, regarding the methods, can have an important consequences in other medical disorders associated with stroke:

- falling - patients with visual disabilities have and increased risk to fall with the most severe consequences as is hip fracture (due to advanced age and associated osteoporosis). Improvement of visual function, physical therapy and adjuvant medication as is vitamin D supplementation can significantly reduce the risk for falling and for its complications (48).

-cognitive disorders - cognitive impairment is a frequent condition associated with stroke patient due to patient's age and due to other associated diseases (49). Visual improvement therapy can also improve cognition due to increasing the patient's ability to repeat the their daily task (50).

- depression: over than $30 \%$ of the patients who have a low vision associated with various disorders have been reported to develop depression (51). Improving visual function shown improvement of the depressive symptoms (51).

Therefore, visual rehabilitation therapies added to other therapies $(52,53,54)$, can constitute and important aid for post stroke rehabilitation patients.

\section{Conclusions}

Visual disabilities after stroke were considered to be irreversible. The researches in this area started to report significant result after various therapies that can prove a considerable potential for vision restoration even in adulthood. Visual rehabilitation after cerebral injury associated with a stroke are usual incomplete but can constitute an important component of stroke rehabilitation method that can lead to a better result in motor recuperation. The strategy for visual rehabilitation therapy has to be adapted to every patient situation and also has to be multidisciplinary. Visual rehabilitation is necessary to be aimed on the improvement of patient's daily life after stoke and constitute a gold target for clinicians who contribute to stroke rehabilitation. Early diagnosis of visual disabilities can substantial improve functional ability and other general aspects in stroke survivors. Post stroke rehabilitation is a activity of a multidisciplinary training that can offer a better social and psychological integration for the surviving patients.

\section{Declaration of conflict of interests}

The authors declare that there is no conflict of interest regarding the publication of this paper.

\section{References}

1. World Health Organization, International Classification of Impairments, Disabilities and Handicaps (ICIDH): A Manual of Classification Relating to the Consequences of Disease. Geneva: World Health Organization, 2002.

2. ICO "Visual standard-Aspect and ranges of vision loss" in International Council of Ophthalmology Report (Sydney), 2002.

3. Hepworth L, Rowe FJ.Visual impairment following stroke - the impact on quality of life: A systematic review. Ophthalmology Research: An International Journal. 2016; 5(2): 1-15.

4. Bulboacă AE, Bolboacă SD, Stănescu IC, Sfrângeu CA, Porfire A, Tefas L, Bulboacă AC. The effect of intravenous administration of liposomal curcumin in addition to sumatriptan treatment in an experimental migraine model in rats. Int J Nanomedicine. 2018; 25;13:3093-3103. doi: $10.2147 /$ IJN.S162087.

5. Kunzmann J, Wolf H, Oberndorfer S. Generalised reversible encephalopathy syndrome: a variant of posterior reversible encephalopathy syndrome (PRES). BMJ Case Rep. 2015; 26; pii: bcr2015210498. doi: 10.1136/bcr-2015-210498.

6. Khan S, Leung E, Jay WM. Stroke and visual rehabilitation. Top Stroke Rehabil. 2008; 15(1):27-36. doi: 10.1310/tsr1501-27.

7. Rowe FJ. Stroke survivors' views and experiences on impact of visual impairment. Brain Behav. $2017 ; 7(9)$ :e00778. doi: 10.1002/brb3.778.

8. Wang TJ, Wang $\mathrm{IJ}, \mathrm{Hu} \mathrm{CC}$, Lin HC.Comorbidities of dry eye disease: a nationwide population-based study. Acta Ophthalmol. 2012; 90(7):663-8. doi: 10.1111/j.1755-3768.2010.01993.x. 
9. Hofer SB, Mrsic-Flogel TD, Bonhoeffer T, Hübener M. Prior experience enhances plasticity in adult visual cortex. Nat Neurosci. 2006;9(1):127-32.

10. Wandell BA. The neurobiological basis of seeing words. Ann N Y Acad Sci. 2011; 1224:63-80. doi: 10.1111/j.1749-6632.2010.05954.x.

11. Orban GA · Higher order visual processing in macaque extrastriate cortex. Physiol Rev. 2008; 88(1):59-89. doi: 10.1152/physrev.00008.2007.

12. Goddard E. A step toward understanding the human ventral visual pathway, J Neurophysiol.2017 ;117(3):872-875. doi: 10.1152/jn.00358.2016.

13. Grill-Spector K, Malach R.The human visual cortex. Annu Rev Neurosci. 2004;27:649-77.

14. Sabel BA, Kasten E. Restoration of vision by training of residual functions. Curr Opin Ophthalmol. 2000; 11(6):430-6.

15. Sabel BA, Henrich-Noack P, Fedorov A, Gall C. Vision restoration after brain and retina damage: the "residual vision activation theory". Prog Brain Res. 2011; 192:199-262. doi: 10.1016/B978-0444-53355-5.00013-0.

16. Frolov A, Feuerstein J, Subramanian PS. Homonymous Hemianopia and Vision Restoration Therapy. Neurol Clin. 2017; 35(1):29-43. doi: 10.1016/j.ncl.2016.08.010.

17. Quint DJ, Gilmore JL. Alexia without agraphia, Neuroradiology, 1992; 34(3):210-4.

18. Unzueta-Arce J, García-García R, LaderaFernández V, Perea-Bartolomé MV, Mora-Simón S, Cacho-Gutiérrez J. Visual form-processing deficits: a global clinical classification. Neurologia . 2014 ; 29(8):482-9. doi: 10.1016/j.nrl.2012.03.006

19. João RB, Filgueiras RM, Mussi ML, de Barros JEF. Transient Gerstmann syndrome as manifestation of stroke: Case report and brief literature review. Dement Neuropsychol, 2017; 11(2):202-205. doi: 10.1590/198057642016dn11-020013.

20. Bruyer R. Color agnosia: a brief review. Acta Psychiatr Belg. 1977; 77(3):309-38.

21. Meyniel C, Bodaghi B, Robert PY .Revisiting Vision Rehabilitation. Front Syst Neurosci. 2017; 11:82. doi: 10.3389/fnsys.2017.00082.

22. Kind PC. Cortical plasticity: is it time for a change? Curr Biol. 1999;9(17):R640-3.
23. Kasten E, Poggel DA, Müller-Oehring E, Gothe J, Schulte T, Sabel BA. Restoration of vision II: residual functions and training-induced visual field enlargement in brain-damaged patients. Restor Neurol Neurosci. 1999;15(2-3):273-87.

24. Narayanan D, Cheng H, Tang RA, Frishman LJ. Optom Vis Sci, Longitudinal Evaluation of Visual Function in Multiple Sclerosis. 2015 Oct;92(10):976-85. 10.1097/OPX.0000000000000684.

25. Dundon NM, Bertini C, Làdavas E, Sabel BA, Gall C. Visual rehabilitation: visual scanning, multisensory stimulation and vision restoration trainings. Front Behav Neurosci. 2015,;9:192. doi: 10.3389/fnbeh.2015.00192.

26. Martins Rosa A, Silva M. F, Ferreira S, Murta J, Castelo-Branco M. Plasticity in the human visual cortex: an ophthalmology-based perspective. Biomed. Res. Int. 2013:568354. 10.1155/2013/568354.

27. Grunda T, Marsalek P, Sykorova P. Homonymous hemianopia and related visual defects: Restoration of vision after a stroke. Acta Neurobiol Exp (Wars). 2013;73(2):237-49.

28. Bulboacă A, Nicula C. Arterial hypotension-risk factor in nonarteritic anterior ischemic optic neuropathy. Oftalmologia.2002; 53(2):52-5.

29. Rowe F, VIS Group UK. Symptoms of strokerelated visual impairment. Strabismus, 2013; 21(2):150-4. 10.3109/09273972.2013.786742.

30. De Haan GA, Melis-Dankers BJ, Brouwer WH, Tucha O, Heutink J. The Effects of Compensatory Scanning Training on Mobility in Patients with Homonymous Visual Field Defects: A Randomized Controlled Trial. PLoS One. 2015; 10: e0134459 doi: 10.1371/journal.pone.0134459

31. De Haan GA, Melis-Dankers BJ, Brouwer WH, Tucha O, Heutink J. The Effects of Compensatory Scanning Training on Mobility in Patients with Homonymous Visual Field Defects: Further Support, Predictive Variables and Follow-Up. PLoS One. 2016; 11(12):e0166310. doi: 10.1371/journal.pone.0166310.

32. Trauzettel-Klosinski S, Rehabilitation of lesions in the visual pathways, Klin Monbl Augenheilkd. 2009; 226(11):897-907. doi: 10.1055/s-00281109874. 
33. Sabel BA, Kruse R, Wolf F, Guenther T. Local topographic influences on vision restoration hot spots after brain damage, Restor Neurol Neurosci. 2013; 31(6):787-803. doi: 10.3233/RNN-139019.

34. Sabel BA, Kenkel S, Kasten E. Vision restoration therapy (VRT) efficacy as assessed by comparative perimetric analysis and subjective questionnaires. Restor Neurol Neurosc, 2004;22(6):399-420.

35. Glisson CC. Capturing the benefit of vision restoration therapy. Curr Opin Ophthalmol, 2006, 17(6):504-8.

36. Mueller I, Mast H, Sabel BA. Recovery of visual field defects: a large clinical observational study using vision restoration therapy. Restor Neurol Neurosci. 2007; 25(5-6):563-72.

37. Hamel J, Kraft A, Ohl S, De Beukelaer S, Audebert HJ, Brandt SA,. Driving simulation in the clinic: testing visual exploratory behavior in daily life activities in patients with visual field defects, J Vis Exp. 2012; (67):e4427. doi: $10.3791 / 4427$.

38. Passamonti C, Bertini C, Làdavas E. Audio-visual stimulation improves oculomotor patterns in patients with hemianopia. Neuropsychologia. 2009, 47(2):546-55. doi: 10.1016/j.neuropsychologia.2008.10.008.

39. Bolognini N, Rasi F, Coccia M, Làdavas E. Visual search improvement in hemianopic patients after audio-visual stimulation. Brain. 2005, 128(Pt 12):2830-42.

40. Frassinetti F, Bolognini N, Bottari D, Bonora A, Làdavas E. Audiovisual integration in patients with visual deficit, J Cogn Neurosc. 2005, 17(9):1442-52.

41. Frassinetti F, Pavani F, Làdavas E. Acoustical vision of neglected stimuli: interaction among spatially converging audiovisual inputs in neglect patients. J Cogn Neurosci. 2002, 14(1):62-9.

42. Pisella L, Mattingley JB. The contribution of spatial remapping impairments to unilateral visual neglect, Neurosci Biobehav Rev. 2004, 28(2):181-200.

43. Koelewijn T, Bronkhorst A, Theeuwes J. Attention and the multiple stages of multisensory integration: A review of audiovisual studies. Acta Psychol (Amst). 2010; 134(3):372-84. doi: 10.1016/j.actpsy.2010.03.010.

44. Cavanaugh MR. Huxlin KR. Visual discrimination training improves Humphrey perimetry in chronic cortically induced blindness. Neurolog. 2017 May 9;88(19):1856-1864. doi: 10.1212/WNL.0000000000003921.

45. Trauzettel-Klosinski S. Current methods of visual rehabilitation. Dtsch. Arztebl. Int, 2011; 108: 871-878. 10.3238/arztebl.2011.0871

46. Peli E. Field expansion for homonymous hemianopia by optically induced peripheral exotropia. Optom Vis Sci. 2000;77:453-464.

47. Lane AR, Smith DT, Ellison A, Schenk T. Visual exploration training is no better than attention training for treating hemianopia. Brain. 2010, 133:1717-1728.

48. Shen SH, Huang KC, Tsai YH, et al, Risk analysis for second hip fracture in patients after hip fracture surgery: a nationwide population-based study. J. Am. Med. Dir. Assoc. 2014; 15:725731. 10.1016/j.jamda.2014.05.010

49. Bulboacă AE, Bolboacă SD, Bulboacă AC, Prodan CI. Association between low thyroidstimulating hormone, posterior cortical atrophy and nitro-oxidative stress in elderly patients with cognitive dysfunction. Arch Med Sci. 2017; 13(5):1160-1167. doi: 10.5114/aoms.2016.60129.

50. Fukuoka H, Nagaya M, Toba K, The occurrence of visual and cognitive impairment, and eye diseases in the super-elderly in Japan: a crosssectional single-center study. BMC Res. Notes. 2015; 8:619. 10.1186/s13104-015-1625-.

51. Nollett CL, Bray N, Bunce C, Casten RJ, Edwards $\mathrm{RT}$, et al. Depression in visual impairment trial (DEPVIT): a randomized clinical trial of depression treatments in people with low vision. 2016, Invest. Ophthalmol. Vis. Sci. 57: 42474254. 10.1167/iovs. 16-19345.

52. Bulboacă AE, Bolboacă SD, Bulboacă AC, Ethical considerations in providing an upper limb exoskeleton device for stroke patients. Med Hypotheses. 2017 Apr;101:61-64. doi: 10.1016/j.mehy.2017.02.016.

53. Dogaru G, Ispas A, Bulboaca A, Motricala M, Stanescu I. Influence of balnear therapy at Baile Tusnad on quality of life for post-stroke patients. Balneo Research Journal. 2017; 8(4): 201-2015.

54. Dogaru G, Ispas A, Stănescu I, Motricala M, Molnár Á. A clinical study on the efficacy of natural therapeutic factors in Băile Tuşnad resort. Balneo Research Journal. 2018; 9(2): 76-81 